\title{
CASTILLOS DE AREQUIPA: UN ARTE POPULAR EFIMERO
}

EdmundoMottaZamalloa ${ }^{1}$

\section{INTRODUCCION}

Hablando de la brevedad del tiempo en que suelen manifestarse las fiestas o las expresiones artísticas, distintas -por ejemplo- a la pintura, debemos a Ernst Hemingway ,el notable novelista y reflexivo observador de los toros, una cabal definición del arte efímero: "los toros son un arte efímero, como el canto y la danza, una de esas artes que Leonardo aconsejaba a los hombresque evitasen; unarteque, cuandoelejecutante ha desaparecido, no existe más que en la memoria de los que lo han visto, y muere con ellos" (1).

Vaya uno a saber por qué razón Leonardo aconsejaba evitar este género de artes. Lo cierto es que a diferencia de las artes perennes, que trascienden al ejecutante y a la memoria del espectador, las efímeras dependen más bien de la presencia real de ambos elementos, atadas a un lapso de tiempo, después del cual sólo queda la sensación de lo ocurrido, cuyo registro se deriva a la memoria, único espacio donde se le puede volver a ubicar. Dentro de la interesante clasificación de las expresiones artísticas,por su duración en el tiempo y en la contemplación, realizada por Teresa Gisbert(2), las fiestas, los duelos, la moda, la comida son artes efímeras tanto como los toros de Hemingway, los fuegos artificiales de todos los tiempos y los castillos de Arequipa de ayer y de siempre.

Siconsideramos el tiempo de permanencia en la contemplación, un castillo se muestra desde el primer

\section{Antropólogo}

encendido hasta el último, en un lapso de seis y diez minutos, como mínimo, hasta quince o veinte minutos, por razones que diremos más adelante. Es durante este breve pero impresionante tiempo que un castillo se presenta como una obra de arte y no antes ni después, en que sólo consiste en una estructura cargada o apagada. Cuando la estructura se desata en luces y movimientos, causando impacto visual en el espectador ysiguiendoun esquema predeterminado porel pirotécnico, recién se puede tener la evidencia de un arte que, gracias a su exhibición, muestra los momentos de goce estético. Poresto, un castillo es el más representativo de las artes efímeras.

Las nuevas tierras de esta parte del continente acogieron a los castillos junto con el insumo del arcabús, se apropiaron yenriquecieron con elementos provenientes de sus propios recursos, invención y creatividad. Hoy podemos hablar de castillos que identifican a diversas localidades. Por lo que toca al Perú, son famosos los castillos del norte -Piura, Cajamarca, Ancash-, Lima en el centro, Ayacucho y Arequipa en el sur.

Arequipa es, quizás, el centro de producción de castillos más importante del sur yacaso del país, porque es aquí donde se queman el mayor número de castillos, y también se encuentra el mayor número de pirotécnicos. Pero sobre todo, porque en Arequipa los castillos se nos revelan muy ligados a las expresiones religiosas, al punto de haber definido su armadura en la forma de una torre compuesta en base a cubos de perfecta cuadratura que ramatan en una cúpula, semejante a la de un campanario. Como afirmara VladimiroBermejo, porboca de uno de sus personajes, en la novela Pólvora: "la pirotecnia tenía 
(...) una categoría estética; tradicionalmente de padres a hijos se iban transmitiendo los secretos de la manipulación de la pólvora y de los explosivos, esmerándose en fabricar acabadas obras de arte"(3).

\section{FENOMENOLOGIA DE LA VISPERA}

Hablar de castillos equivale a hablar de la víspera. Ocurre al comenzar no una noche de cualquier día, sino del que precede a la fecha central, aquélla que viene con misa de fiesta, salva de troya a la hora de elevar el cáliz, con procesión de pompa por las arterias principales del pueblo, corso de bellezas y rosadas alegorías, que han transformado la plaza pública o el atrio de la iglesia en un bullicioso escenario.

Oficiosos en el arte de la pirotecnia han vestido el castillo, antes que termine el día,colocándolo en el atrio del templo,convertido desde ese momento en el protagonista de la noche, pues lo que vendrá enseguida sólo está en relación a la quema de castillos. Mientras terminan de vestir el castillo se fueron agregando al contexto dianeras con sus hervidores de bebidas para conjurar el frío,entre los que no se puede dejar de mencionar el té pitiáu, una infusión para machos,por lo cargado de pisco o aguardiente que contiene; o en las vecindades hacen humear crepitantes visceras sobre brasas de carbón;anisado de nájar o cualquier licor que haya alcanzado el prestigio de bajamar, ingredientes vitales de la fiesta tanto como la pólvora o la vihuela de otros tiempos conqueacompañabanuna pampeña.Como puede ofrecer su testimonio, ya no un historiador como Ventura y Travada, ni Echevarría y Morales, sino un octogenario que conserva aún fresco el pasado reciente dirá, sin reservas éticas ni titubeos históricos:"no hay fiesta en Arequipa sin música, castillos y trago". Tratándose de si la víspera del patrono o de la santa celebradaydellugardonde sellevea cabo,enelempedrado piso del atrio de la provincia, o en la polvosa plaza de un distrito rural, se encienden los tercios de queuñal o yareta llevados en vigorosas mulas de arriería para evitar la agonía del mundo por enfriamiento. Los amplificadores dispersan sentidas melodías del yaraví, marineras y alegres pampeñas en las que no falta la presencia de cuerdas y ejecutantes que representan a esa música genuina y campestre que devuelve a la memoria una historia traída a retazos.

A breves intervalos, que se reducen a medida que transcurren las horas, se sienten en el espacio nocturno, estallidos de cohetes que anuncian la proximidad de los castillos.

Una profunda vocación colectiva se expresa durante las celebraciones. Gentes de diversa procedencia que se han resistido a permanecer en la tibieza de sus casas, acuden al escenario como si se tratase de un llamado de la sangre.Los pasos se desplazan sin rumbo fijo, aunque hay un solo propósito que los ha animado a asistir: los castillos. Verlo es un gozo, no importa que caigan sobre sus hombros fragmentos de carrizo o partículas de ceniza candentes. No habrá de durar mucho, es cierto, pero el poco de tiempo que estará ante la mirada es suficiente. La fiesta delfuego no puede tener mejor expresión que a través de esa carnatura que son los castillos. Cuando la primera crepitación se anuncia con un breve estallido en su base tras el incendiario cigarrillo con que el maestro transmite fuego a la guía, se sienten exclamaciones, gritos, ojos en movimiento para no perderse algo de ese juego de luces que se desplaza porel largo de la armadura de cañabrava. Laemoción irá en aumento,conforme las luces van recorriendo cada uno de los cuerpos del castillo hasta desbordarse en los momentos siguientes en que, tras el vuelo de las palomas, la iluminación cubre toda la armadura o hace resaltar en ella la imagen del patrono del pueblo. Es como si el castillo hubiera dado alcance a su destinatario,como una manda de fuego que, pirotécnico mediante, el pueblo pío, a través del devoto,enviara para conseguir ese momento perdurable de goce, pero sabiendo que se ha cumplido de todos modos con un deber religioso.

\section{EL FUEGO Y LA FIESTA}

Desde sus inicios la pirotecnia (del griego pur, fuego y tekhne, arte) juntó las necesidades de la guerra con las de la fiesta. Los principios de aplicación a los usos de la guerra fueron los mismos que asistieron a los fuegos artificiales.

Los pueblos asiáticos manifestaron, en todo tiempo, gran entusiasmo por el arte del fuego para los festejos nocturnos, empleando preparaciones de iluminación, quemando mezclas incendiarias y lanzando artificios de fuego al espacio.La profunda afición de chinos, indios y egipcios se transmitió a los griegos y romanos que dejaron descripciones de fiestas nocturnas en que el arte del fuego constituía el ingrediente más importante. Ya se empleaban entonces sustancias diversas como elaceite de nafta, mezcladocon diferentes resinas, grasas y sustancias minerales, causando preocupación entre los fabricantes el olor que tales sustancias desprendían y el humo que se formaba.

Tras la derrota de los romanos, la pirotecnia sufrió una notable decadencia que se prolongó hasta los siglos XIy XIlen que resurgió con innovadas composiciones. El gran impulso que la pólvora dió al uso de la pirotecnia en la guerra y en la fiesta, hizo que los árabes detonaran en España imponentes juegos artificiales con bombas, cohetes, tracas y luces de colores, que hicieron exclamara un viejo cronista: "no había festejo popular en 
que no se corriera la pólvora".

El gusto por las luminarias fiestas fue trasladado a otros países por aragoneses y catalanes. La acogida no se hizo esperar en Italia y Francia, alrededor del siglo XV, paises donde, después de un prolongado paréntesis en que el progreso de la pirotecnia se volvió a estancar, resurgió con vitalidad a mediados del XIX con el empleo de nuevos agentes químicos como el clorato de potasa y el nitrato de estroncio, además de otras sustancias entre combustibles y comburentes, algunas de comportamiento especial como las limaduras de hierro, de acero o de zinc, y otras que sirvieron para comunicar a la llama una coloración determinada en combinación con sales de estroncio y cal.

Su producción en lberoamérica se ha identificado con una afición cada vez más creciente, al tiempo que se fue consolidando en un elemento esencial de la víspera en las fiestas patronales y aniversarios cívicos.

Es de imaginar el asombroyadmiración con que los naturales del nuevo mundo, Méjico y Perú, sucesivamente, recibieron los fuegos artificiales y los castillos, en contraste con el temor que sintieron ante la pólvora reventada por los arcabuceros.

Los primeros castillos que se vieron en Lima y otras nacientes ciudades coloniales, los hacian, tal vez, empleando un material distinto a la cañabrava o carrizo, antes de recurrir a este elemento como base de su estructura.

La gran tradición que existió y existe, hoyen día, se debe a su cultivo a partir de la Colonia. Algunos pirotécnicos gustan distinguir etapas históricas, tomando como referencia la armadura. De acuerdo con esta apreciación particular, los castillos habrian pasado del palo de pájaro bobo al de la cañabrava, y de este último a una reciente estructura metálica, todavía en experimentación.

No estamos en condiciones de afirmar como fueron los castillos hechos conel palo de pájaro bobo (4), pero si podemos decir que fueron utilizados en la confección de castillos durante los primeros años de la Colonia, antes de que se empleara habitualmente la cañabrava (phragmites communis), o bambú, una planta rizomatosa que crece a lo largo de ríos, riachuelos,canales de regadío, cuya producción es abundante especialmente en los valles de la costa y lugares cálidos de la entrecordillera,además de ser común en todo el mundo (5). Su empleo es muy diverso como material de construcción y de confección de canastos, pero por su forma entubada y hueca no se dejó esperar su aplicación en castillos y fuegos artificiales.

Atendiendo a la estructura, está por iniciarse la etapa siguiente a causa de la necesidad planteada de reutilizar la armadura en un mercado, a la vez tradicional y creciente.El metal facilita el reuso, pero tambien contribuye significativamente al ahorro de trabajo y su consiguiente costo, y de tener que aprovisionarse periódicamente de los valles productores. Algunos pirotécnicos han visto con muy buenos ojos las ventajas del metal, al punto de pensar seriamente en abrir una sección de soldadura en su taller.

Existen también otros criterios para pensaren la evolución de los castillos. Aparte del material de que están hechos,se incluye la energía en uso, sola o combinada.De acuerdo con este criterio de clasificación se pueden distinguir castillos criollos, eléctricos y los de control a distancia. Los dos últimos pueden denominarse modernos, que se diferencian de los criollos por el hecho de que al castillo tradicional se le agrega dispositivos eléctricos, de modo que el mensaje que el castillo desea comunicar permanezca iluminado por un tiempo determinado. Pero también, el uso de conductores eléctricos se hace atractivo por la facilidad que confiere el hecho de accionar a distancia el encendido de cohetes y candelillas.

Las innovaciones han introducido cambios singulares desde los años 1958-59 en lo que se refiere a la confección de castillos. Dicha diferencia se hace notoria a partir de 1965 en que se puede hablar de castillos modernos. Sin embargo no se debe generalizar estas innovaciones, comositodos los pirotécnicos se acogieran a la idea de modernizar sus trabajos, ni mucho menos que se haya dado en todo el país. Depende mucho del interés puesto por los propios pirotécnicos, quienes gustan de diferenciarse entre sí por el grado de profesionalismo con que se dedican a la pirotecnia. Desde este punto de vista, algunos pirotécnicos se dedican a su oficio más por la necesidad de subsistencia que, pordecir lo menos, el amor al oficio. El resultado del trabajo dedicado al arte del fuego difiere de un pirotécnico a otro.Como bien lo grafica uno de los pirotécnicos dedicados a la producción de castillos por amor al arte, los "otros", maestros o no, se encuentran dispuestos a reproducir estereotipos conocidos, o introducen los cambios sólo cuando han sido comprobados.

En base a sus propias declaraciones podemos distinguiren qué etapa se encuentran los pirotécnicos en la producción de castillos. Algunos preferirán no experimentar con cables eléctricos, porque demanda esfuerzo o constituye, apenas, un elemento decorativo. ¿Que han cambiado a partir de 1958? Nazario Hernani dirá, tomando como referencia sus propios trabajos, que ha dado un mayor tratamiento a una sola cara de las cuatro que tiene un castillo, con el propósito de conferir 
una más acabada expresividad en su composición. Por otro lado, el cambio comprende el uso de cohetes y chispas, los que son de mejor confección, y llevan incorporados, dispositivos eléctricos en los castillos. Aunque es notorio también, que la referencia a una nueva etapa tiene que vercon los iniciados experimentos de las estructuras en base a metal. Hay que mencionar que junto a los medios y mecanismos tradicionales, manipulados en la preparación y combinación de material inflamable, se utilice el reciente empleo de la computadora con el propósito de mejorar el control de la calidad de los insumos.

\section{FAMILIA Y TRADICION}

La pirotecnia es un oficio de familia, en parte porque se ha heredado de los padres y de los abuelos, en una tradición que puede remontarse a los antepasados de los tiempos de la Colonia. La tradición explica la pervivencia de una vocacióny de los secretos volcados en la pirotecnia, un conjunto de saberes que es necesario resguardarcomo marca de calidad, garantía y distinción. $Y$ esto se consigue solamente, si se encierran en el círculo familiar los menesteres que exigen la producción de fuegos artificiales (6), en especial cuando se trata de conservar las fórmulas en la obtención de colorantes, pues de eso depende en gran medida la originalidad en los efectos de iluminación. No es extraño hallar en la familia del pirotécnico un hermetismo absoluto a la hora de hablar sobre colores y colorantes.

La producción artesanal de castillos se realiza con cierto grado de tecnificación alcanzado en su factura. Por razones de seguridad, los talleres pirotécnicos se encuentran en la periferie de la ciudad, aunque el centro de operaciones, tanto para la construcción de castillos como de las tareas menores que no impliquen riesgo alguno, se encuentran en la propia vivienda del maestro.

Un taller promedio se compone de secciones, de acuerdo con los requerimientos que demande la construcción de castillos: el lugar de molienda de la pólvora,con uno o varios batanes; el lugar de confección de las armaduras en base a cañabrava; el lugar de cargar las "mechas" o "chispas"; el lugar de confeccionarlas guias; aparte del depósito de cañabrava.

Además del maestro, que es el cabeza de familia, intervienen los hijos varones yocasionalmente la esposa. Por tradición participan los varones. Este es un arte reservado para el sexo fuerte; por lo demás no se ha oído hablar de pirotécnicos mujeres. Para la mujer existen otras tareas, en especial para la esposa, quien asume la representatividad de entablar los compromisos, o para realizar actividades consideradas al margen de la pirotecnia, pero que de un modo u otro tienen que ver con ella.

Los hijos varones empiezan desde pequeños en el oficio de pirotécnico, realizando tareas que le permitan su edad. No pocas veces el niño de 12 o 14 años es todo un maestro.

El trabajo de construir un castillo supone categorías y tareas especializadas que se distribuyen del siguiente modo:

Maestro : muele la pólvora. Es de cuidado y prefiere hacerlo personalmente.

Obrero : hace los cuerpos.

Casquillero : hace los casquillos, sin cargarlo, en especial los de troya.

Moledor de

pólvora : reemplaza eventualmente al maestro en molienda de pólvora. (Hay especialistas para los 48 talleres).

Cordelillero : tarea que incluye la confección de casquillos, candelillas y guías.

Torcedor

de figuras : a cargo del maestro en colaboración de los demás miembros del taller. Hacen el arte completo y miden la velocidad.

Operario : es el que arma el castillo.

A esta actividad circunstcrita a la familia, suelen agregarse eventualmente personas cuyo concurso es requerido libremente por los 48 talleres que existen en Arequipa (7), quienes pueden acceder al talleren calidad de obreros $u$ operarios. Un aspirante a maestro debe atravezarpor etapas. "Tendrá que serbueno, muy bueno, en otras palabras ser de la familia para confiarle todo. Si no lo es puede hacer figuras, cohetes, hacer el castillo; pero jamás se le daria la fórmula de los colores".

Sin embargo, la necesidad de incrementar la producción para abastecer una creciente demanda en el sur del Perú, y en ocasiones excepcionales por los países vecinos,los talleres de pirotecnia, en especial aquellos que tienen un mercado reconocido, se ven obligados a tomar por contrato mano de obra ajena a la familia. Con mayor razón los que han conseguido asentar su plaza en ciudades y pueblos apartados de la costa y de la sierra, requerirán el concurso de ayudantes con cierto conocimiento en los riesgos de la pirotecnia. Aunque cada maestro tiene una plaza ganada donde ha logrado asentar sus reales, no significa que esté negado el ingreso a su eventual dominio de otro cuyo arte acaba de ser reconocido al paso de su prestigio, pudiendo así desplazar a los anteriores. De alli el gran interés dedicado a obtener una buena performance, que repercutirá en la demanda sobre su trabajo, pues los devotos son los clientes principales. Como los castillos van asociados a las festividades religiosas, son los devotos quienes al asumir el cargo de alferados o mayordomías de la fiesta 
los que se convierten en los verdaderos críticos de su arte,y quienes decidirán, obedeciendo a una manda de devoción, la construcción de un castillo.

\section{VESTIR EL CASTILLO}

A primera vista la armadura de los castillos del norte o centro del pais tienen diversas formas -los hay trapezoidales, triangulares, rectangulares-, compuestos por cuerpos cuyo número y altura son variables (8); y desde el punto de vista de su exposición pueden consistir en uno o más de uno, apareciendo como un conjunto disgregado, en el que uno de ellos puede ser el castillo principal. Aqui se trata de varios castillos más que de uno solo. En cambio, los de Arequipa han logrado estandarizarse en el número de unidades, número de cuerpos otiempos, en las figuras o motivos representados y hasta en la ubicación de estos en los castillos. Y como está visto, hasta en la forma. Los castillos de Arequipa nos dicen cuan juntos están de las expresiones religiosas, pues se han logrado cuajar en la forma de una torre. Sus cinco cuerpos son cuadrangulares, o cubos de lados iguales, y tamaños iguales, y una cúpula o coronación que termina en un vértice, exactamente como rematan las torres de una iglesia (ver esquema).

Se da por cosa establecida que esta estructura no puede admitir alteraciones, digamos porque opera como un esteoreotipo. Resulta inimaginable un castillo de tres cuerpos o uno de seis. Aun por razones de economía los castillos, por simples o "vistosos" que puedan ser(9), respetarán siempre la estructura clásica de cinco cuerpos o tiempos.

La estructura o armadura de un castillo está compuesta de cuerpos, o, como prefieren denominar los pirotécnicos, de tiempos. La razón por la cual se designan con el nombre de tiempo a un cuerpo del castillo es porque está identificado con el momento o etapa de los encendidos.

El primer tiempo es todo un subsistema dentro del conjunto. Comprende a su vez instancias concatenadas, al cabo del cual sobreviene una suerte de paréntesis,sin luces ni explosiones,salvo el humo y el olor ácido que se dispersa por el ambiente, puntos que señalan precisamente que se acaba de concluir con la composición del primer tiempo. El espectador a captado en este primer tiempo el juego de luces y movimientos que describen la forma de una "S" (de allí el nombre de "eses"). El segundo tiempo está dominado por el movimiento horizontal de los "piriguchos" que, al igual que las eses, son en número de cuatro. El tercer tiempo está dedicado a los rombos, también en número de cuatro, los que describen un movimiento horizontal. Mucho depende del ingenio del pirotécnico la forma de

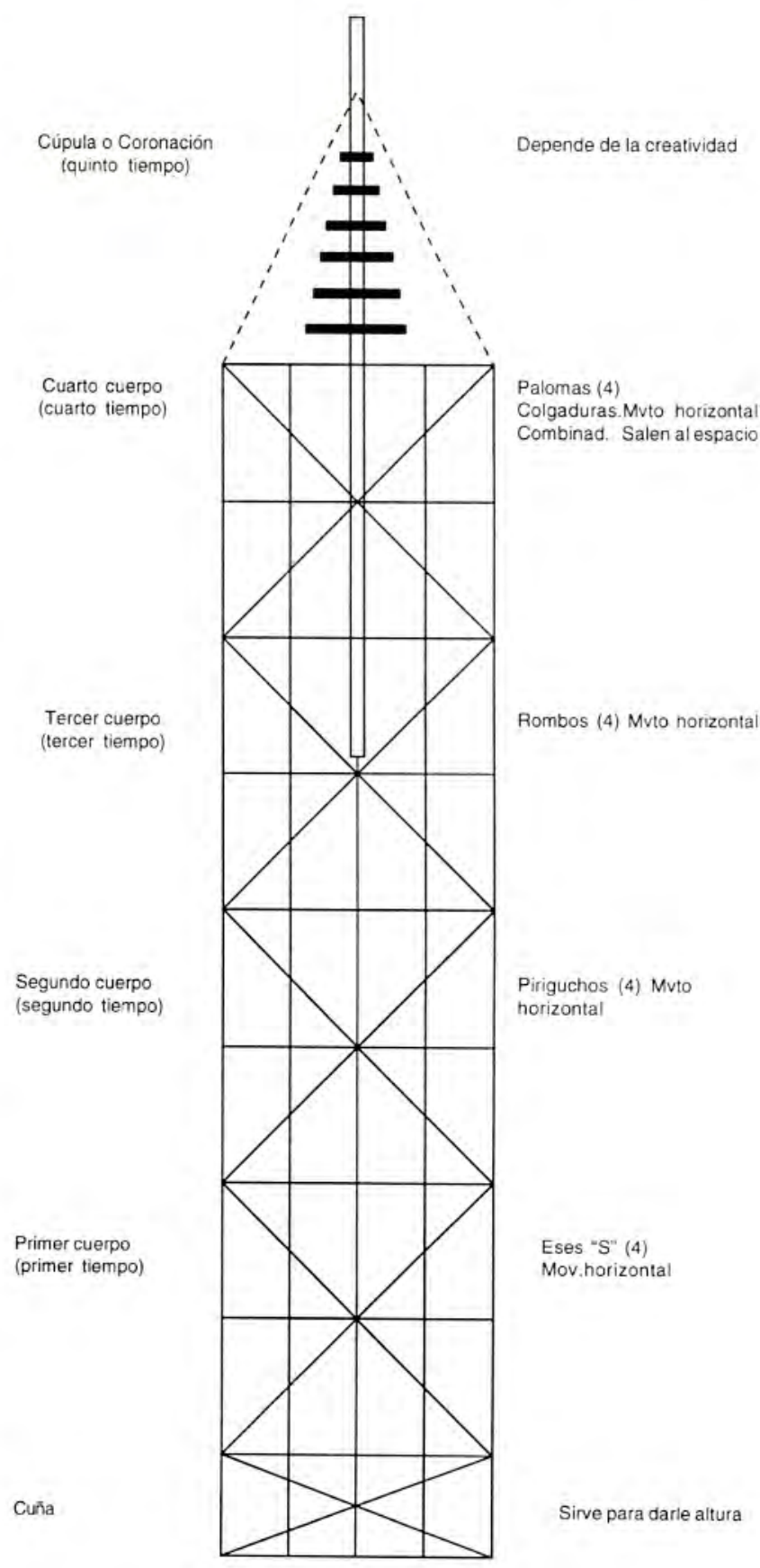

paso de un tiempo a otro, y también la articulación de ellos al finalizar uno de los superiores. El cuarto tiempo puede presentar en el cuerpo colgaduras, diseños circulares, repetir eses, poner en movimiento conos alargados ("piriguchos"), pero el estereotipo que gobierna la presentación de este cuerpo son las "palomas" que, por turno, sucesiva o simultaneamente salen al espacio como coronas alargadas con un relente de luces.Un espectador acostumbrado a apreciar los castillos de Arequipa sabe poranticipado las figuras que el pirotécnico 
ha considerado en cada uno de los tiempos, lo que no sabe es la composición que aparecerá ante sus ojos.

En el quinto tiempo, de la coronación, o la cúpula, el despliegue técnico, de imaginación, de colory arte corre por cuenta de la creatividad del pirotécnico. Los motivos varían respondiendo a su inspiración yel mensaje que desea comunicar, pues como todo artista, el pirotécnico utiliza la composición para transmitir algo o presentar de una manera singular el motivo de la festividad cuya víspera se está celebrando. En la práctica, el dominio que el pirotécnico alcance en la coronación se expresa en el éxito del castillo, y,en definitiva, de su obra. Algunos gustan presentar tras una aureola de luces figuras que representan al volcán Misti, la papaya arequipeña, la estrella, los mundos, los corazones, que al ser accionados realizan movimientos que van parejo al despliegue de los colores. Este define el momento culminante del castillo, pues durante ella hay pirotécnicos que suelen hacer aparecer a la Virgen u otra figura simbólica, motivo de la fiesta, a quien está consagrado el castillo.

La estructura de cuatro cuerpos, más la cúpula, aparte de la cuña o cuerpo de menor tamaño que sirve sólo para dar altura al conjunto, es común a los castillos de Arequipa, al igual que los elementos estereotipo cuya factura y empleo conoce todo pirotécnico.Y no se ha podido otorgar mejor denominación que el de "vestir el castillo"al hecho de disponer en los cuerpos las figuras y demás elementos que involucran guias, chisperos y candelillas(10).

El trabajo de la armadura incluye los cuerpos y la cúpula del castillo; incluye también la confección de las figuras hechas a base del mismo material utilizado para los cuerpos (11).

Ensegundo lugar está el trabajo correspondiente al uso de la pólvora para la elaboración de guias y cohetes. En este caso, el primer asunto en cuestión está vinculado con la obtención de la misma pólvora. Los pirotécnicos distinguen dos clases, una fina y otra corriente o chusca. La corriente se obtiene mezclando azufre, salitre y carbón, que se muele en un batán dispuesto para el caso. Esta clase de pólvora es destinada para las troyas. La pólvora fina se obtiene de mezclar molidos de carbón fino, nitro y aluminio, y es la que se destina preferentemente para los castillos. Después de la molienda viene el cargado en los casquillos de cañabrava observando la proporcionalidad que deberán guardar el peso yla medida con el tamaño y velocidad que se desea imprimira las figuras. La velocidad es efecto de la relación proporcional entre peso y tamaño del chispero (cohete). Un chispero (del normal que se emplea) mueve 2 kilos o 2,5 kilos de peso. Para 1 kilo bastará un chispero "chico y delgado". Una chispa normal pesa poco más de una onza. Para mover 2 kilos se necesita una onza de chispa. Ciertamente que a más chispa aumenta la velocidad y con ella el efecto visual.

Antes de empezar a "vestir" el castillo,el pirotécnico debe tener la seguridad del rendimiento de su trabajo, pudiendo para ello someter a prueba reiteradas veces el comportamiento de alambres y focos ;cuántas revoluciones por minuto para aumentar la fuerza de la pólvora. Las pruebas se pueden realizar por partes, sin empleo de máquinas; basta la pericia de prenderlo suspendiendo la figura. Por cuestión de perfeccionamiento, la atención del pirotécnico está concentrado en la relación entre las revoluciones por minuto y el grosor del cohete, empleando para aprobar su experimento sus dos únicos sentidos: la vista y el oído.

Este trabajo corre a cargo del maestro debido a su alto riesgo, y porque haciéndolo él mismo, puede asegurar el efecto que desea conseguir.

Las guias constituyen otro elemento que requiere del empleo de la pólvora. Dependiendo de la complejidad y del costo de un castillo, el número de guias puede variardesde 10015 hasta 20 o más. De longitud variable, se extiende del nivel de la cuña hasta enlazar el chispero de la figura que pondrá en acción. Las guias son cubiertas con papel y con el cuidado que no debe sufrir ninguna mella y de ese modo en lugar de encender la figura indicada, de acuerdo al plan del maestro, terminan encendiendo otras figuras de la estructura.Cada tiempo requiere de tantas guias cómo encendidos deberán hacerse. El encendido de la composición de juegos que se presenta los hace el propio maestro, o alguien designado especialmente por él, desde el pie del castillo o ubicado a una prudente distancia. Algunos pirotécnicos están deseosos de sustituir las guias tradicionales por conductores alámbricos, los que podrían ser accionados mediante batería; pero este hecho aún se encuentra en una fase experimental.

El costo de este trabajo,a la vez familiar y artesanal, deberá asumir el piadoso devoto cuya promeza incluye quemar uno o más castillos en la víspera del santo patrono del pueblo. De él tanto como de la pericia del pirotécnico dependerá la complejidad del castilloyen último término el acabado artístico (12). A la calidad de sus trabajos estarán ligados también la plaza y frecuencia de oportunidades con que reciben los encargos(13). La fama conserva el mercado. Una oportunidad de contrato se da cada vez que se enciende un castillo.Y se considera que un pirotécnico ysu equipo de trabajadores han tenido éxito cuando al finalizar una "quema" reciben contratos para la siguiente festividad. 


\section{ARTE HASTA LAMUERTE}

Cualquiera que haya tenido la ocasión de conversar con uno de esos pirotécnicos entregado a su trabajo de creación, revisando diseños, probando la fuerza de un chispero ola velocidad de una figura, valorará de inmediato la expresión: "este arte es hasta la muerte", como una definición acabada de esa clase de trabajo que absorve el tiempo y la vida enteros.

De otra manera resultará difícil entender las razones que llevan a enfrentar riesgos, no porque se tenga en menos consideración la vida del propio maestro y acaso de la familia,ni siquiera porque en el orden económico no sea tan rentable como puede inferir un espectador al ver en unos cuantos minutos hacerse humo una considerable cantidad de dinero. Tal vez porque el propio riesgo de jugar con fuego que le viene mejor al arte de la pirotecnia y de los fuegos de artificio, revelan en el hombre la atracción del peligro.

Lo más notorio, lo inmediatamente perceptible en los castillos es que se tratan de esa clase de objetos que impresionan la sensibilidad estética, pero que al mismo tiempo denotan conocimientos empíricos e intuitivos de ciencia y técnica; los que se pueden percibir poreldominio de la pólvora en una determinada estructura y por el efecto luminoso que produce una calculada combinación de sales metálicas. Un pirotécnico dirá inmediatamente que en los castillos no sólo hay arte, sino también ciencia y técnica.

Si se siguen los detalles del encendido, secuencia tras secuencia, y al final se observa el conjunto de la composición, no puede estar más claro el hecho de que se trata de una obra cuidadosamente planificada, con precisas determinaciones en su diseño y el cálculo del tiempo que a través de las secuencias nos hace intuir la noción de que el pirotécnico no sólo ha querido mostrarnos una dinámica composición de colores, sino que todo el esquema está orientado a un objetivo, a ese algo que el pirotécnico nos quiere expresar a través del castillo. Después del último encendido el espectador no sólo tiene la impresión de haber visto una escena de luces, sino también cuando menos una vaga idea de su costo económico y de todo el despliegue técnico, artesanalmente elaborado,que significa la producción de esas luces multicolores.

Varios elementos concurren en la determinacion del castillo: composicion, color,sonido y movimiento. Adicionalmente se muestra un fondo necesario sobre el cual es posible apreciar tales elementos en su combinacion y secuencia. La composición está referida a la combinación de figuras, cuyo movimiento yencendido sigue una secuencia por tiempos, comprendiendo desde figuras-estereotipo hasta aquellas que traducen el mensaje y que usualmente son colocados en el tiempo de la coronación.

La primera clase de figuras son empleadas por la mayoría de pirotécnicos y se refieren a las "eses", a los rombos, a las palomas. La segunda se refiere a aquellas figuras que en lo posible son creaciones últimas del pirotécnico, el cual incluye la presentación de elementos que indican el motivo de la festividad, rito o personaje del que forma parte el castillo.

Toda la composición es manejada por el maestro desde el pie del castillo encendiendo sucesivamente, de acuerdo a su diseño, las guias. Cada guia supone un encendido, de uno o parte de un tiempo del castillo. Para un castillo criollo, el número de guias varía de quince a veinte. La mayoría de ellas corresponden a la coronación o quinto tiempo. Cada guia tiene un número determinado de secuencias que tienen intervalos a veces de hasta medio minuto entre una secuencia y la siguiente; esto se debe más exactamente al tiempo que el pirotécnico emplea para poner en acción la siguiente secuencia.

Otro aspecto de la composición se refiere a los colores, o luces de colores, que van asociados a las figuras y secuencias. Estos están dados generalmente por los remates de voladores -puntas de parís, colas, lluvia o cascadas- en las que se suceden los colores desde primarios hasta combinados. Una serie de "etapas" dispuestas dentro del cuerpo del cohete, determina la secuencia de los efectos, mientras que la longitud de las uniones establece el intervalo entre ellos. Al fondo, o sea el primero en la secuencia, está el cohete propulsor que lleva la carga a una altura predeterminada.

Durante el recorrido de ascenso se van encendiendo, una tras de otra, esparciendo su carga con sus efectos. Al explosionar el elemento propulsor tiene el efecto sonoro con algo de humo. Todos los elementos son combustibles. Existe gran variedad de fuegos artificiales y sus formas obedecen a la ocasión, el lugar y la escenografía específica, aunque todos tienen un principio común.

Al hacerlo el pirotécnico sabe exactamente cual será la intensidad de los colores que estallarán en el espacio. Para obtener una mayor visibilidad y precisión de las secuencias tienen presente la necesidad de que dichas sales tengan en lo posible cierta pureza para evitar los humos, cuya reducción tan deseada se consigue mediante el control del azufre y, de alguna manera, recurriendo también a la dirección del viento a la hora de ubicar el castillo. El pirotécnico sabe que todo ello apunta a un solo objetivo: conseguir la máxima vistosidad al menor costo. En esta expresión se encuentra resumida 
toda su concepción de arte. Para esto, además de composición y colores ha empleado el movimiento en la que las figuras se mueven en sentido horizontal,circular, vertical o combinado, especialmente cuando se trata del quinto tiempo. Las figuras no sólo se encienden y explosionan en multitud de colores, sino que describen un movimiento,generando fascinación en el espectador.

En la fabricación yensamblaje de los cohetes se utilizan diversos materiales y técnicas.

Los componentes activos junto con los elementos neutros se constituyen en un dispositivo denominado "candelilla", que por su apariencia semeja a un chispero. Los hay de diversos tamaños, elegidos en función del efecto que se desea obtener. Las candelillas producen las luces, lo que los chisperos las explosiones.

En la composicion hay un elemento asociado que no está en los planes del pirotécnico, pero que se supone es algo explicito: la oscuridad, como un lienzo gigante y negro sobre el cual se despliega la secuencia de figuras y colores. Los castillos se encienden de noche, siempre. Se da por descontado también que debe haber un oportuno pronóstico del tiempo, pues la lluvia es el peor enemigo del pirotécnico(14). Tal vez se deba a esta ultima razón el hecho de que en lugares lluviosos los castillos han tenido un menor desarrollo.

Otro elemento no menos importante se relaciona con el sonido. En lugares deseados de la secuencia, sepueden agregar ruidos de explosión. Mientras que las partes luminosas -contenidas en envases frágiles-producen un leve sonido al explosionar, la pólvora encerrada en material resistente explosiona con fuerte ruido. Mediante el empleo de cohetes silvadores se consiguen efectos adicionales, pues un zumbido continuo, nítido e intenso es deseable cuando acompaña el vuelo de las palomas, o el estallido de una bombarda. En cambio los ruidos producidos por la crepitación de laguadúa olos borbotones de una mezcla mal calculada deslucen la presentación del castillo,en cuyo caso es preferible reducir a su menor expresión, cosa que por otra parte resulta del todo imposible.

Toda la composición transcurre en un lapso de 10 a 20 minutos, al final del cual el espectador tiene la impresión de haber apreciado un cuadro, una escena que, como todo objeto de arte, puede provocarle agrado o asombro.

El pirotécnico manipula principios y recursos técnicos por intuición. En este sentido es, o debe ser un empírico de la química por excelencia. Pues mucho depende del tipo de pólvora que debe emplear, cantidad que debe incluir en un chispero o cohete asociado a una figura, número de chisperos para determinar una velocidad deseada; y lo consigue mezclando sustancias en peso y proporción precisadas. El pirotécnico trabaja con balanza y no renuncia, en su aprendizaje, a recurrir a medios proporcionados porla modernidad. Otro aspecto relacionado con la química son los colorantes para conseguir tal o cual juego de luces, los que se hace mediante la combinación de sales y sustitutos en fórmulas que se reservan bajo siete llaves con el propósito de mantener una relativa ventaja en la competencia; de igual manera se cuidarán los diseños considerados como exclusivos.

El grado de eficiencia obtenida en la manipulación de componentes del castillo, que se expresa luego en el preciosismo de la presentación, va asociada a la noción de técnica, un término que los pirotécnicos gustan emplear para referirse a sus trabajos. El empleo de mecanismos e instrumentos, no siempre convencionales, está sujeto al continuo interés de perfeccionar, en un sentido no sólo mecánico, sino de conseguir, descubrire, inclusive, inventar nuevos mecanismos y elementos que impliquen ahorro a su economía y agreguen un distintivo a su creatividad.Guiado por el objetivo de alcanzarcada vezprecisión yexactituden la presentación de su obra, algunos pirotécnicos no han dudado en recurrir a conductores de energía eléctrica. En este sentido, la definición que sobre su técnica ha expresado el maestro N. Hernani, es elocuente: "si una figura está hecha para diez encendidos, pues debe encenderse en diez veces y no en ocho ni en nueve".

\section{DIALOGODE LOS COLORES}

La vistosidad perseguida por el pirotécnico es el resultado de una cuidadosa combinación de agentes químicos,activos y neutros,con una clara noción de contraste y luminosidad que deben ocurrir durante el despliegue de secuencias, en la que cumplen adicionalmente un papel decisorio los intervalos. La armonia, el brillo y el contraste de los colores no harán otra cosa que resaltar el diseño de los estereotipos contenidos en los tiempos del castillo, pero también darán cuenta de una específica escenografía al desatarse libremente en el espacio nocturno. El efecto de conjunto suele ser cualificado por el propio pirotécnico como una conversación de los colores, acaso en referencia inequívoca a una lograda armonía.

En la conversación polícroma, el juego de luces yel efecto de voladores son dos aspectos fundamentales en los castillos y fuegos de artificio, los mismos que se obtienen mediante la composición de sales metálicas colocadas en materiales "portadores" que,al alcanzar altas temperatuas, se encienden dentro del cohete 
durante el tiempoy dirección predeterminados.El principio físico operante, igual al de un tubo fluoroscente o una estrella, es la emisión de energía electromagnética, que, mediante la explosión del activo químico, produce la emulsión sucesiva de colores: el bario produce luz verde, el calcio violeta, el cobre azul,el sodio amarillo, el estroncio rojo. Altiempo que los tonos e intensidades se obtienen variando la granulación de las partes activas que llevan los compuestos metálicos.De ese modo aparecen ante el espectador simples chorros de explosión instantanea hasta una escenografía de colores y formas que dependen de la disposición de los compuestos.

El delicado balance de los componentes, tanto en el aspecto técnico como en la composición química, es el producto de una pericia empírica que transmite la experiencia yel secreto familiar otorgado porla tradición. Se conocen las virtudes cromáticas del cobre, del estroncio, del sodio,del calcio y del aluminio, así como las tonalidades a que darán origen, pero donde hay diversidad manifiesta es en la formulación de las combinaciones aliadas con materiales neutros, lo mismo que en la disposición y los tiempos establecidos del encendido que forman parte delatesoramiento individual.

Diálogo de colores o sinfonía polícroma, hecho de fuego y pólvora, acompañada de notas explosivas, ocupan en su despliegue un espacio que ha sido igualmente pensado durante el diseño del castillo ,a saber por la amplitud y altura en que tendrán lugar la explosión de colores así como la expansión de los efectos de voladores, todo lo cual repercutirá en la visibilidad y despliegue de las formas.

Los remates de los voladores se obtienen con la misma lógica que las luces condicionadas dentro de las "candelillas", que no son otra cosa que pequeños cohetes de etapas. Variando la proporcionalidad de los componentes activos se configuran en el espacio estreIlas, cabelleras, subida de chispas, paracaídas, truenos, culebrillas y los efectos en estrellas simples 0 compuestas. Con las limaduras de alumnio y hierro se reproducen efectos de chispas, o algo más espectacular como una "lluvia" de estrellas. Con aluminio fino puede formar primorosas cascadas que llegan a rebotar del piso; mientras que con aluminio oscuro sólo se consigue una caída de luminaria cabellera que se disuelve a cierta altura. En su interés por reducir el costo de un castillo, relacionado con el empleo de las sales metálicas, que son las de mayor demanda en la producción de colores, los experimentados pirotécnicos pueden incluir elementos variantes como la glucosa o la leche en polvo. En lugarde utilizar un kilo de nitrato de estroncio para obtener el rojo, agregan 12 onzas de glucosa con el cual se obtiene un rojo intenso, efecto que puede hacer pensar que se ha empleado el equivalente de 1,5 kilos de nitrato de estroncio.
$\mathrm{Y}$ asi por el estilo otras modificaciones que son el resultado de la experiencia.

El interés por la policromía no consiste sólo en saber cómo se obtiene el azul o el verde, sino el verde llevau o la rosa bajita, es decir, en conseguir las tonalidades del mismo color, de modo que la secuencia de las formas sean correspondidas con una escala cromática cuando el movimiento circular pasa a una espiral, o en su defecto degrade un tono intenso a medida que disminuye de velocidad un estereotipo, pues así como agrada el contraste también gusta la gradualidad del color. No es de otra manera como el entusiasmo febril del maestro hace exclamar en el momento de profusión de luces,movimientos y colores: "el rojo debe conversar con el azul, el azul con el verde, el verde llevau con el amarillo".

\section{DELESPIRITU BARROCO}

Por lo demás y tal como ocurre con los objetos que tienen origen en el trabajo artesanal, un castillo "sirve' y "dice" algo. En el primer caso se trata de un objeto destinado para la conmemoración. Por consiguiente, tiene el estatuto de un objeto ritual, su función es equivalente al de una cera encendida en el altar de una imagen sagrada y que en si mismo responde a una "manda": aquello que se destina en cumplimiento de la promeza o, por el contrario, constituye el recurso de una petición. En una palabra, se trata del motivo que lleva a presentarel castillo. Lo sabremos cuando en determinada secuencia de la composición aparece iluminado en la cúpula un recuadro conteniendola imagen del destinatario (santo patrono ola del héroe cívico o militar), que per-dura en la contemplación breves segundos. Ciertamente, el mensaje no depende sólo del pirotécnico. Como su trabajo es resultado del encargo (salvo en los concursos pirotécnicos), el mensaje es sugerido por el devoto. El pirotécnico recibe del cliente las sugerencias que desea ver en el castillo y, naturalmente, esta petición está sujeto al precio que permite la capacidad económica del mandante; y el resto del trabajo corre por cuenta del maestro quien sabe por anticipado que su libertad creativa está condicionada por el motivo de la presentación sugerida; pero que, pese a las dos limitaciones, se convierten en un desafío para la imaginación, pues, al fin y al cabo, nadie interrogará por el precio del castillo, ni siquiera cuestionará el motivo, sino que se limitará a restringir un calificativo en términos tan simples como "estuvo muy bonito"o "falta experiencia", o premiará con aplausos o simple y llanamente con el movimiento breve pero significativo de la cabeza. Esto ocurre cuando el espectador ha pasado por ese instante que se denomina " momento culminante del castillo", al finalizar la coronación. 


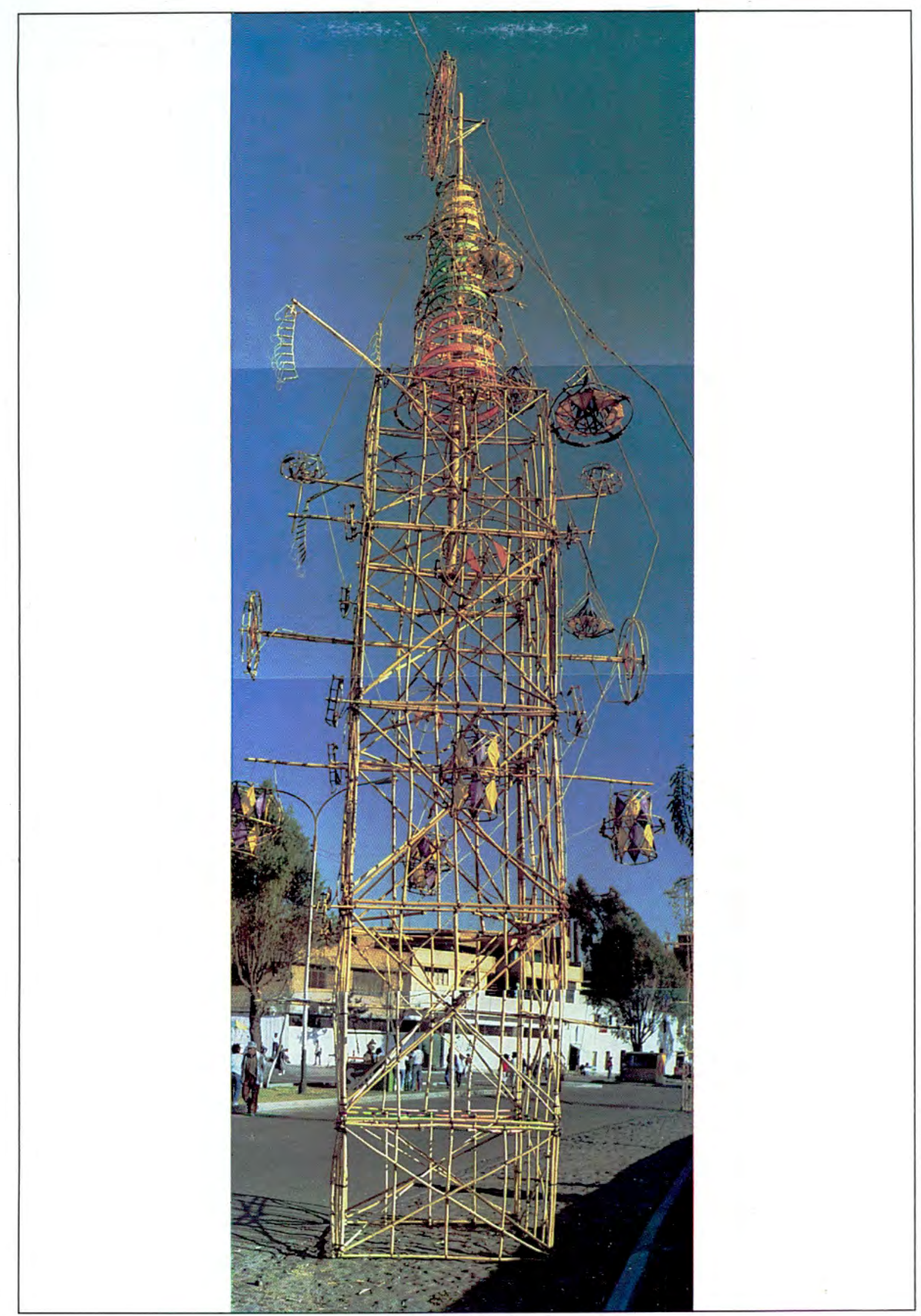

"CASTILlo vestido" 


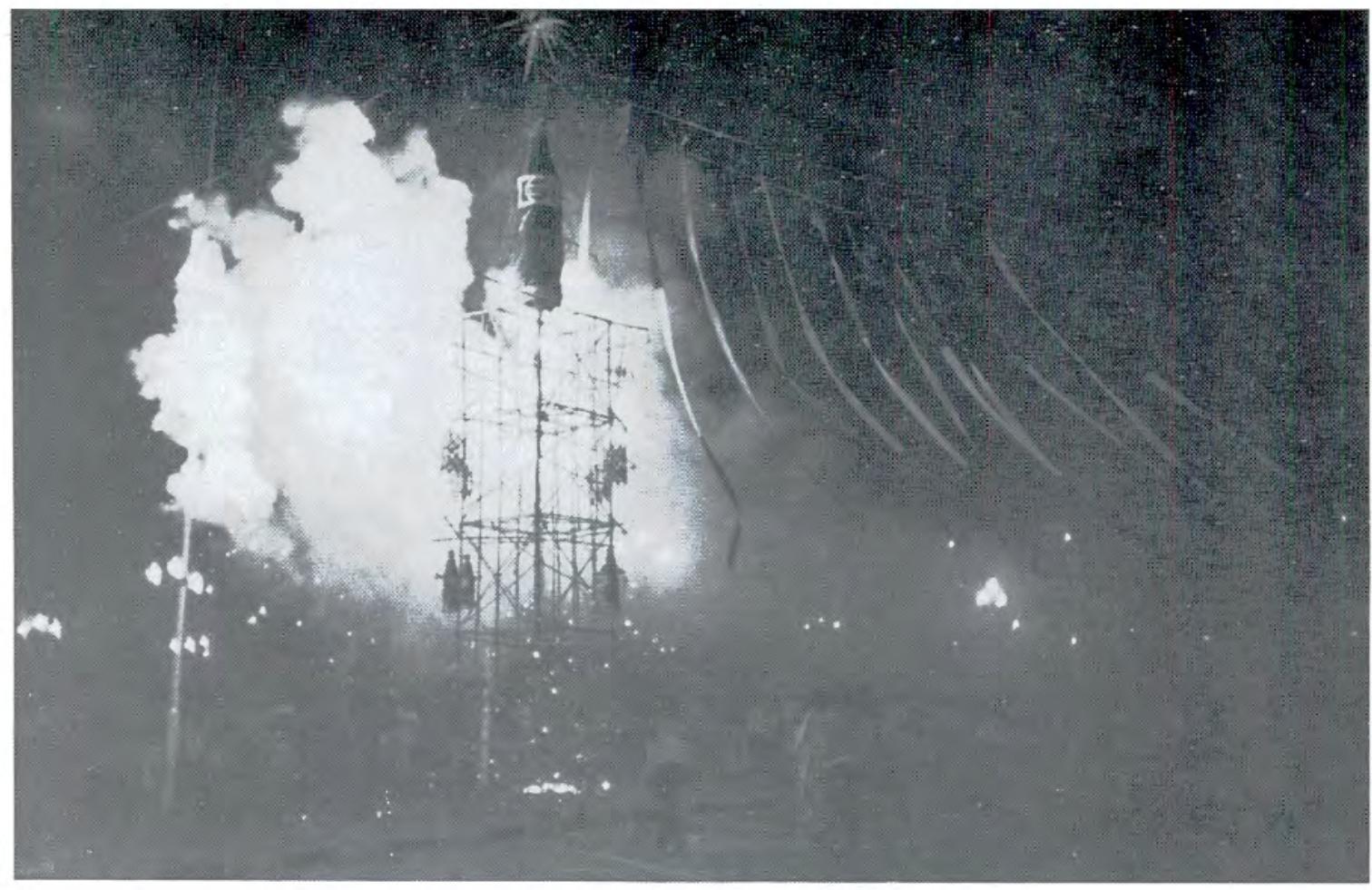

Un comercial asociado a símbolos de países latinoamericanos, prueba de que los castillos son un excelente medio de publicidad.

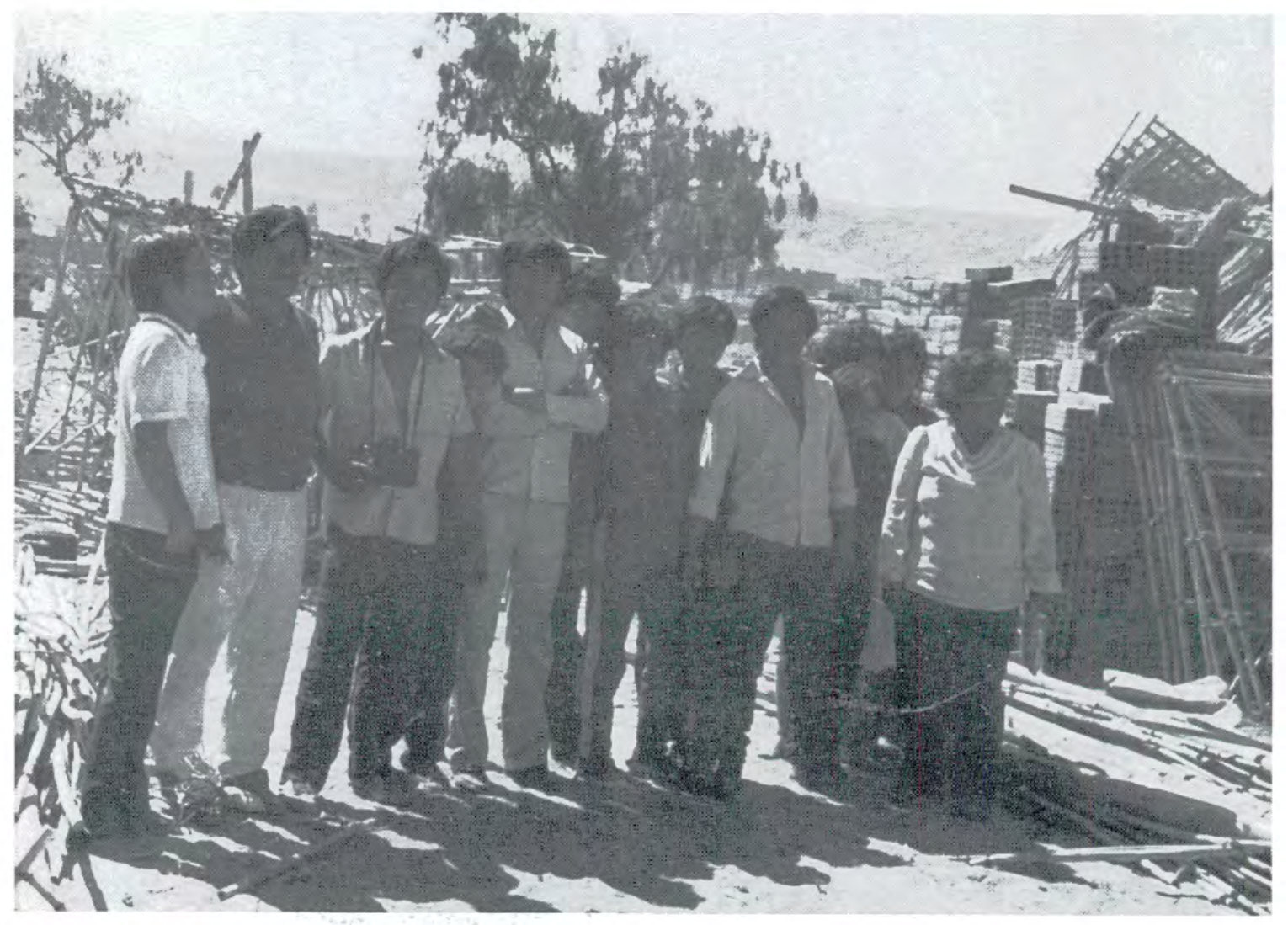

El Maestro y su familia en un trabajo que es preferentemente familiar. 
Si lo barroco es un estilo que recoge la ornamentación caracterizada por la profusión de adornos,en la que predomina la linea curva,el color primario y altisonante, el derroche de imaginación y riqueza en la expresividad de las formas, un castillo, como en general los fuegos artificiales, obedece efectivamente a un barroquismo de espíritu que pretende mostrarse de manera abierta, desenfadada y colorida. EI espíritu barroco no tiene necesariamente una delimitación histórica, en el sentido de que se le pueda identificar exclusivamente con el siglo xv o xviii, ni es exclusiva a europa, ni a México o Perú de los inicios de la colonia; se trata antes bien de una expresión cíclica que tiende a retornar en la historia, y más propiamente es un fenómeno que suele suceder a los momentos de honda crisis anímica y social de los pueblos. Pero también forma parte, de manera permanente, de las expresiones expansivas que se corresponden con los momentos previos a las circunstancias plena y manifiestamente festivas.

Los castillos son apenas una muestra del espíritu expansivo del arequipeño. Expresan una potencial creatividad popular marcada a fuego por el sentimiento religioso que ha inspirado su arquitectura. A través de los castillos, mucho más que a través de losfuegos artificiales, el arequipeño de raigambre hispana y mestiza hace conocer su vocación de barroquismo, incorporando en el conjunto de sus expresiones estéticas y culturales, aquella forma muy particular del ser popular.

\section{NOTAS}

(1) E. Hemingway: Muerte en la tarde.

(2) En:La fiesta: arte efímero. El Mercurio, Chile, 1984.

(3) En Pólvora, novela costumbrista arequipeña.

(4) Esta especie no tiene caña hueca, pero sirvió probablemente parala armaduraporsus tallos relativamente largos y resistentes.

(5) Véase el trabajo "La vegetación desértica perenne..." deZegarra,Rosario. Nueva Imagen Nro.5,1994.

(6) Es el caso de los hermanos Hernani, quienes encuentran su sello de distinción en sus antepasados. Todos están dedicados a la pirotecnia, uno de ellos no hadudado de abandonar sutítulo de contadorpara abrazar el llamado de los ancestros, al igual que los hijos decididos como todo artista a vivir para su arte y de su arte. No los amilana ni los riesgos ni accidentes; a diferencia de otros pirotécnicos que no dudarían un instante de abandonar la pirotecnia si consiguieran otra ocupación razonablemente rentable,porque la pirotecnia expone sus vidas frecuentemente a los riesgos y accidentes, que no son pocos, desde luego, y que han llegado a comprometer inclusive la propia vida. Nombres de familias dedicadas a la pirotecnia más populares son ,aparte de los Hernani, los Alvarez, los Chávez, cada una de ellas reconoce a un tronco común, a partir del cual los descendientes han formado sus propios talleres.

(7) De los 48 talleres, 37 están registrados en el padrón de la Asociación de Pirotécnicos, yestos en conjunto, en el Ministerio de Industrias y Turismo, e igualmente en el Ministerio del Interior. Once talleres están en situación de clandestinidad.

(8) Se hablan de castillos que tienen hasta 20 metros de altura, y compuesto de 8 cuerpos o más, como ocurre en el norte.

(9) En su propia percepción, los pirotécnicos suelen denominar "baratos" a los castillos simples, y "caros" a los vistosos.

(10) Hay que resaltar que en la construcción de un castillo han intervenido varios hombres, bajo la dirección del maestro. Algunos maestros se ven obligados, dependiendo de la magnitud del castillo, la importancia de la festividad y el número de castillos encargados, emplean hasta doce hombres.

(11) La cañabrava identifica a los castillos prestándole un tono artesanal. Su producción está difundida en los valles de Majes, Vitor y Tambo.

(12) Los precios de los castillos se localizan entre los 400 y 2000 nuevos soles (180 y 900 dólares respectivamente). Un costo promedio ofertado para las festividades se ubica alrededor de 700 nuevos soles. En relación a su costo, los castillos pueden clasificarse en pobres, normales y caros. Pobres son los castillos cuyo costo se ubica por debajo, aunque no a significativa distancia, de los 400 nuevos soles. La experiencia recomienda al pirotécnico deshechar todo compromiso que suponga un costo más económico pues atentaría contra su prestigio, que a los ojos del espectador acabaría siendo deslucido. En tales casos, el maestro aconseja al cliente reemplazar el castillo por juegos artificiales. Por debajo del precio limite "ya no sería un castillo", pues sería demasiado pobre, y un castillo demasiado pobre no existe. En cambio, un castillo normal tiene un precio reconocido en el mercado,y su ligera variación, de uno a otro pirotécnico, se debe a su prestigio. Estos son los castillos que tienen mayor demanda, y los devotos pudientes pueden encargar la construcción de uno 
o más castillos, dependiendo en todo caso de su capacidad económica. Por otra parte, los castillos caros, cuyo precio puede sobrepasar incluso los 2000 nuevos soles, son encargados para ocasiones especiales, cuando se tratan de festividades de gestion corporada y de amplia repercusión social. Se incluyen también en esta categoría los castillos confeccionados para eventos de competencia regional o nacional en las que el pirotécnico, corriendo con los costos por riesgo y cuenta propia, debe presentar un despliegue espectacular. Pero también pertenecen a esta categoría los castillos encargados para su presentación en el extranjero,

\section{BIBLIOGRAFIA}

Bermejo, Vladimiro

1948 Pólvora; novela, Arequipa.

Espinoza S.,Waldemar

1987 Artesanos, transacciones, monedas y formas de pago en el mundo andino, siglos $X V$ y XVI; T.I, Banco Central de Reserva del Perú.

Echeverría y Morales,Fco.

1946 (1804) Memorias de la Santa Iglesia de Arequipa. En: Memorias para la historia de Arequipa, de V.Barriga.

García Canclini, Néstor

1982 Las culturas populares en el capitalismo. Casa de las Américas, La Habana. como ocurre algunas veces con pirotécnicos cuyos trabajos son reconocidos en paises como Brasil, Argentina o Chile.

(13) Algunos queman a razón de uno o dos castillos por semana, otros uno por cada mes.

(14) El temor a la lluvia que puede frustrar la presentación de castillos y repercutir en una pérdida económica, ha sido un reto al ingenio del pirotécnico. Los Hernani, por ejemplo, han recurrido a cubiertas de plástico para no interrumpir la fiesta delfuegoaunen plena llovizna, debiendo para ello retirar la cubierta simultaneamente al hecho de activar las guías.

Hemingway, Ernest

1985 Muerte en la tarde. Seix Barral.

Unger, Tomás

1984 Losfuegos artificiales. En: El Comercio,28 de agosto.

Lauer, Mirko

1982 Crítica de la Artesanía. Desco, Lima.

Sánchez Moreno,V.

1987 Arequipa colonial y las fuentes de su historia, Lima.

Valdivia, Juan Gualberto

1847 Fragmentos para la historia de Arequipa.

Lechuza

de arenal 